\title{
CLINICAL CASE WITH IMMEDIATE PLACEMENT OF IMPLANTS AND EARLY LOADING WITH MOVABLE PROSTHETIC STRUCTURE OF A PATIENT WITH GENERALIZED PERIODONTITIS AND II-III DEGREE MOBILITY OF THE TEETH
}

\author{
Georgiev T., Nogalchev K. \\ Department of Oral and Maxillofacial Surgery, \\ Department of Prosthetic Dental Medicine, \\ Faculty of Dental Medicine, Medical University - Varna, Bulgaria
}

\begin{abstract}
:
One of the most common inflammation disease in the oral cavity for the past few years is the chronic form of parodontitis. Every 2nd or 3rd patient who has been to the dentist has the illness. If a person has this illness he cannot have an implant treatment done, because it is temporarily contraindicated until the oral hygiene and status is restored. Patients with reoccurring chronic periodontitis, who need an implant treatment are a real challenge for the dental surgeon and the prosthetis.
\end{abstract}

Treatment of patients with defects in teeth rows with the help of intrabone implants may be considered as one of the greatest achievements in modern dental medicine. The concept of teeth implants originated in the beginning of our era and became immensely significant after the 80 s of the previous century with the acknowledgment of osteointegration.

Here are some of the most important issues, which are still subject of a heated discussion:

- When is the implant to be placed in the alveolus of the extracted tooth;

- When is the implant to be loaded?

The method regarded as traditional is the one in which it is waited for three to six months for the alveolus to heal after extraction of the tooth and application of the two-stage method of implantation, i.e. after the implant is placed, it is initially sutured for a period of three to four months when in the lower jaw and for five to six months when in the upper jaw, thus waiting for the complete osteointegration of the implant. Afterwards the implant is uncovered and a healing abutment is placed thus shapinges the gum above the implant, while after two to three weeks the temporary structure is replaced by e permanent one.

The problem with the traditional method is the fact that for rehabilitation of patients who haves lost their tooth/ teeth it has to be waited for approximately six to twelve months.
At a later stage, however, the results of experimental research showed that failure to load the implant for prolonged periods is not a prerequisite for a successful implant treatment and that osteointegration is, de facto, also possible with in shorter healing periods and even after immediate loading of the implant.

Nowadays, there are four different methods of placement of implants applied, depending on the post extraction period:

1. Immediate placement - waiting period of zero days, i.e. it is placed immediately after tooth extraction;

2. Early placement - waiting period of four to eight weeks, it is related to the healing period of the soft tissue around the tooth alveolus;

3. Early placement with partial bone healing - twelve to sixteen weeks;

4. Late placement - the traditional method involving a six-month waiting period.

When we talk about distal teeth, i.e. the patient does not feel discomfort from an aesthetic point of view, the traditional method, which has been best studied and involves the least number of risks, is to be preferred. However, if the patient in question has generalized periodontitis with teeth mobility of II-III degree, which is not subjected to treatment, and her job depends on their looks, then the attending doctor has to choose between risk and tradition.

\section{CLINICAL CASE STUDY}

A patient, age 55, was admitted to the Department of Oral and Maxillofacial Surgery with complaints of a periodic pain in the teeth related to their often inflammation, mobility of the teeth of the upper jaw $(14,13,12,11,21,22)$, bleeding gums and inability to chew. The medical history reveals that the missing dentition was lost as a result of gradual loosening of the teeth one after another, with their subsequent extraction. Clinical examination reveals mobility of the upper jaw teeth $(14,13,12,11,21,22)$, oedematic and inflamed papillae with discharging of drops of pus, upon 
probing in the subgingival space - sharp pain, depth of periodontal pockets - over $10 \mathrm{~mm}$.

The patient was prescribed antibiotic treatment. After finishing the antibiotic course of treatment, it was noted that the symptoms of acute inflammation were no longer present, however, the oedematic papillae and the II-III degree mobility had not disappeared.

While considering different options for treatment, we decided on tooth extraction with subsequent implant insertion and a removable prosthesis on them.

\section{COURSE OF SURGERY:}

Under local infiltrative anesthesia with Mepivastesin $3 \% 4 \mathrm{ml}$ in the region of teeth $14,13,12,11,21,22$, a mucoperiosteal flap was formed carefully by means of a periotome, additional horizontal incisions were made along the crest of the alveolar ridge respectively behind 14 and 22 teeth. The teeth were carefully extracted; the wound was cleaned from any granulations and washed with a solution of Hibiscrub, Hydrogenii peroxidatum 3\% and physiological solution. Implants were placed in the region of the alveolar ridge of tooth 15 , in the alveolus of 13 and 22, as well as in the region of 23. The choice of selection of positioning the implants was driven by the presence of preserved alveolar bone and by the desire of the patient not to have an augmentation of the alveolar ridge and guided tissue regeneration. By placing the implants, initial stability was achieved (the implants were screwed in, self-tapping). Therefore, it was decided to leave open the implants with placed healing abutments. The post-surgical period was without complications. The patient was examined every day in order for the surgical field to be washed and for the prevention of complications of inflammatory nature.

\section{ORTHOPEDIC TREATMENT:}

After placing the dental implants, provisional removable dentures were made which have free spaces in the healing abutments. After positioning the tooth implants, provisional removable dentures with cleared beds in place of the healing abutments were prepared.

After removing the sutures and the healing of the gingival, removable dentures were prepared on the basis of a standart prosthetic protocol: taking of an anatomical impression from the prosthetic area on the basis which individual spoons for the patient were prepared; taking of functional impression from the prosthetic area by means of open transfers; determining the central position of the lower jaw; test with arranged teeth; transfer on the prostheses. Because of the accurate transfer of the position of the upgrades at the laboratory, the prostheses were completely finished with parts of the spherical upgrades attached to them.

The post operational period was without complications. The patient was prescribed an everyday secondary check-up with the intention of sanitizing the operated area and prevention of complications of an inflammatory character as well as providing advice for maintenance of the oral hygiene around the implants.

Subsequently clinical examinations after the first, third, and sixth month were prescribed as well as after the first, second and third year in order to assess the stability of the implants and monitoring possible inflammation around them. Clinical parameters under examination:

- Plaque Index (PI);

- Bleeding on Probing (BOP);

- Periodontal Decease Index (PDI);

- Gingival Index (GI);

- Feeling of pain and discomfort;

- Mobility of tooth implants;

- Complications with prosthesis above implants;

- Paraclinical parameters;

- Orthopantomogram before the beginning of the operation; after placing the implants; after first, second and third year.

Table 1.

\begin{tabular}{|l|r|r|r|r|c|}
\hline Assessment Indexes & $3 \mathrm{~m}$ & $6 \mathrm{~m}$. & $12 \mathrm{~m}$. & $24 \mathrm{~m}$. & $36 \mathrm{~m}$. \\
\hline Bleeding on Probing (BOP) & $12,5 \%$ & $6,25 \%$ & $6,25 \%$ & $12,5 \%$ & $6,25 \%$ \\
\hline Plaque Index (PI) & Stage II & Stage I & Stage I & Stage II & Stage I \\
\hline Gingival Index (GI) & Stage 0 & Stage 0 & Stage 0 & Stage 0 & Stage 0 \\
\hline Periodontal Decease Index (PDI) & Stage 0 & Stage 0 & Stage 0 & Stage 0 & Stage 0 \\
\hline Pain Sensation & - & - & - & - & - \\
\hline Mobility of Tooth Implants & - & - & - & - & - \\
\hline Prosthesis Problems & - & - & - & - & - \\
\hline
\end{tabular}




\section{RESULTS DISCUSSION:}

Results from the clinical research showed that, at a minimum of three years clinical use, removable dentures over implants are an acceptable and predictable method of a prosthetic recovery.

Immediate placement of implants after the extraction of periodontally problematic teeth, if the operational area has being thoroughly sanitized before and during the operation, does not constitute a problem for a consequent orthopedic recovery.

The clinical result gives an opportunity for additional clinical research of the same type in order to further clarify the loading indication of immediately placed implants after the extraction of teeth with periodontal problems.

This clinical case gives the opportunity to presume that the effectiveness of the orthopedic treatment may be the same both to patients with and without periodontal

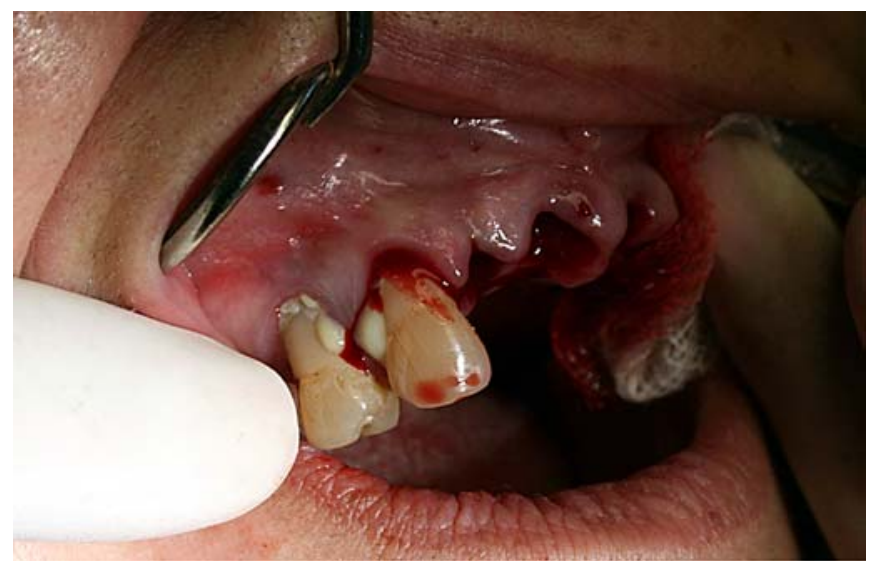

Pic. 1.

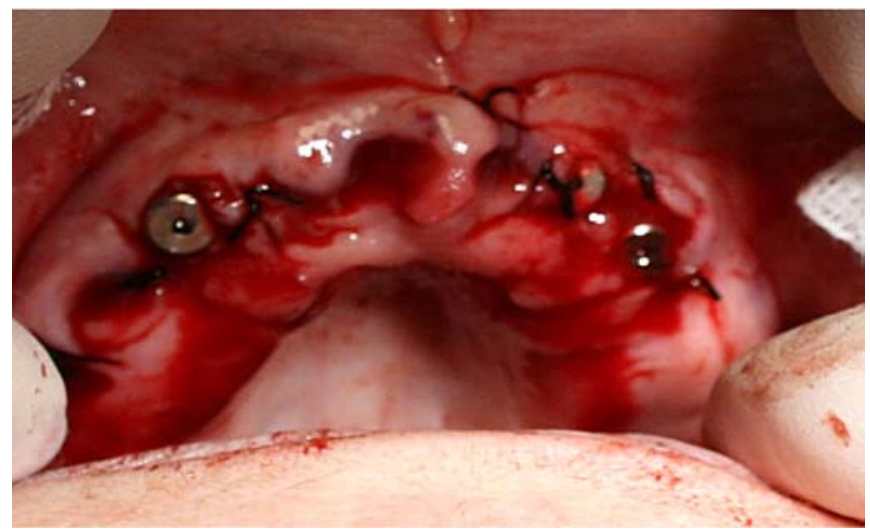

Pic. 2. pathology.

The authors of this article believe that the following factors are of utmost importance when dealing with similar clinical cases:

- Chirurgical aseptics and antiseptics

- Achieving primal stability when placing the implant

- Making of temporary dentures during the period of healing of the mucous membrane

- Strict control and education of the patient in personal oral hygiene

\section{CONCLUSION:}

The result of the conducted treatment shows that, over the course of a minimum of three years, such treatment may lead to a predictable result with patients who lost their teeth as a result of periodontitis. This itself is a prerequisite for further deepening of the research on the topic.

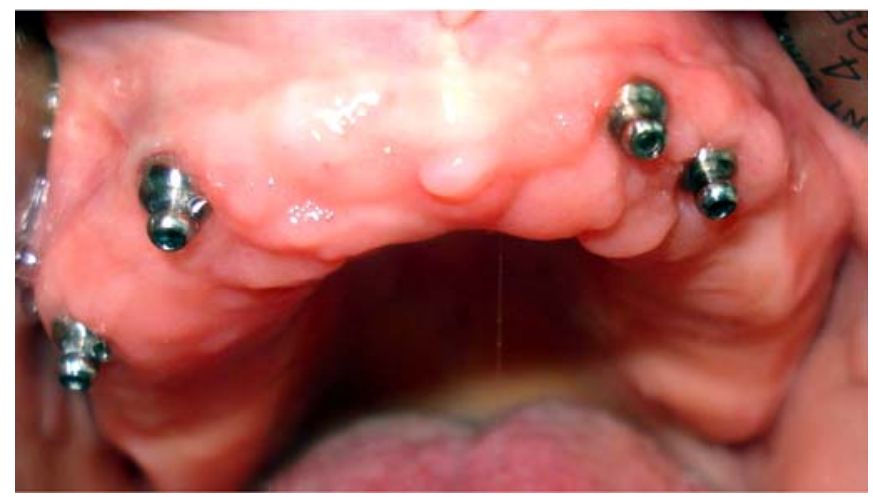

Pic. 3.

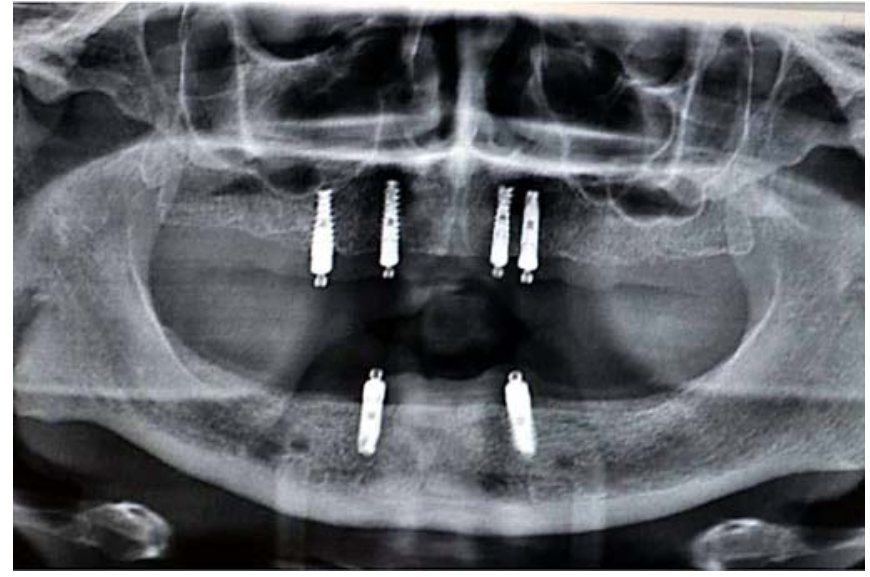

Pic. 4. 


\section{REFERENCES:}

1. A. Stavropoulos, J. Nyengaard, N. Lang, T. Karring (2008) Immediate loading of single SLA implants: drilling vs. osteotomes for the preparation of the implant site. Clin. Oral. Impl. Res. 19, 2008/ 55-65

2. Szmuckler-Moncler, S.Salama, H. Reingeweritz, Y. \& Dubruille, J.H. (1998) Timing of loading and effect of micromotion on bone-dental implant interface: review of experimental literature. Jouranl of Biomedical material Research 43:193-203.

3. Schitman, P.A., Wohrle, P.S. \& Rubenstein, J.E. (1990) Immediate fixed interim prostheses supported by two-stage threaded implants: methodology and results. Journal of Oral Implantology 16: 96-105.

4. Tarnow, D.P., Emtiaz, S. \& Classi, A. (1997) Immediate loading of threaded implants at stage I surgery in edentulous arches: ten consecutive case reports with 1to 5-year data. International journal of Oral and Maxillofacial Implants 12: 319-324.

5. Paul A. Schinitman, DDS, MS/Peter S. Wohrle, DMD/Jeffrey E. Rubenstein, DMD, MS Immediate Fixed Interim Prostheses Supported by Two-stage Threaded Implants: Methodology and
Results;

6. Dittmar May, DMD, MD, Dr Med/ Georg E. Romanos, DDS, Dr Med Dent Immediate implant-supported mandibular overdentures retained by conical crowns: A new treatment concept;

7. Lyndon F. Cooper, DDS, PhD/ Amin Rahmen, BDS, MPH/John Moriarty, DDS, MS/Nancy Chafee, DDS, MS/Debra Sacco, DDS, MS Immediate Mandibular Rehabilitation with Endosseous Implants: Simultaneous Extraction, Implant Placement, and Loading;
Correspondence address:

Dr Tihomir Georgiev

18 Hristo Popovich St., Varna 9000, Bulgaria

e-mail: tgeorgeivphd@yahoo.com 